\title{
Proceso Formativo de Docentes Universitarios para la Inclusión de Estudiantes con Discapacidad
}

\section{ESJ Social Sciences}

\section{M.A.P.E. Pamela Franco Díaz}

Universidad Autónoma de Chihuahua, México

\section{Rigoberto Marín Uribe}

Universidad Autónoma de Chihuahua, México

\section{Yunuen Soccorro Rangel \\ Ledezma}

Universidad Autónoma de Chihuahua, México

\section{Ma. Concepción Soto \\ Valenzuela}

Universidad Autónoma de Chihuahua, México

Submitted: 22 September 2020

Accepted: 02 November 2020

Published: 30 November 2020

Corresponding author:

Ma. Concepción Connie

DOI: 10.19044/esj.2020.v16n31p61

(c)

Copyright 2020 Ma. Concepción Connie et al. Distributed under Creative Commons BY-NC-ND 4.0 OPEN ACCESS

\section{Resumen}

Las prácticas educativas en el nivel superior exigen calidad dirigida a la diversidad e inclusión, por lo cual, cada vez es mayor la tendencia de estudiantes en la universidad en búsqueda de oportunidades de formación profesional para integrarse a la vida laboral. La inclusión de estudiantes con alguna discapacidad a las aulas universitarias ha tomado por sorpresa a profesores debido las necesidades de estos alumnos, por la existencia de la escasa preparación adecuada para hacer frente al reto, de formar personas dentro de una educación inclusiva. El objetivo de este trabajo es narrar las experiencias vividas, con un grupo de profesores, durante un programa de formación docente orientado al desarrollo de prácticas educativas inclusivas. El estudio es con un enfoque de investigación-acción utilizando la narrativa para la recolección de datos. El programa plantea formar profesores universitarios en la atención a alumnos con discapacidad, con una educación para todos, atención a las diferencias. Participaron diez docentes que imparten clases en grupos que tienen estudiantes con discapacidad (visual y motriz). Los resultados arrojados son: 1) Diseño del programa de formación en el cual se presenta la versión final de los contenidos y ejes de programa y 2) secuencias didácticas (SD) como guía del proceso formativo, donde se muestran las matrices y experiencias de validación de las SD y narrativas de las experiencias del docente. Se concluye que la formación docente constituye un desafío para la universidad al destacar una necesidad profesional de liderar las acciones educativas, relacionadas con la diversidad desde la inclusión.

How to cite this article

Ma. Concepción, C. et al, (2020). Proceso Formativo de Docentes Universitarios para la Inclusión de Estudiantes con Discapacidad. European Scientific Journal, ESJ, 16(31), 61. https://doi.org/10.19044/esj.2020.v16n31p61 
Palabras claves: Formación docente, inclusión, persona con discapacidad, secuencia didáctica

\title{
Training Process of University Teachers for the Inclusion of Students with Disability
}

\author{
M.A.P.E. Pamela Franco Díaz, \\ Dr. Rigoberto Marín Uribe, \\ Dra. Yunuen Soccorro Rangel Ledezma, \\ Dra. Ma. Concepción Soto Valenzuela, \\ Universidad Autónoma de Chihuahua, México
}

\begin{abstract}
Educational practices at the higher level require quality aimed at diversity and inclusion. As a result, the rate at which students in the university search for professional training opportunities to integrate into their work life is increasing. The inclusion of students with a disability into university classrooms has taken teachers by surprise due to the needs of these students. It is also attributed to the lack of adequate preparation to face the challenge of training people in an inclusive education. This paper focuses on narrating the experience lived, with a group of teachers, during a teacher training program aimed at developing inclusive educational practices. The study was performed with an action-research approach using a narrative for data collection. The program proposes to train university professors to pay attention to students with disabilities, through education for all, and also focus on their differences. Ten (10) teachers who taught group of students with disabilities (visual and motor) participated in the study. The results obtained are: 1) Design of the training program in which the final version of the contents and program axes is presented and 2) didactic sequences (SD) as a guide to the training process, where the matrices and validation experiences are shown by the SD and narratives of the teacher's experiences. In conclusion, teacher training constitutes a challenge for the university by highlighting a professional need to lead educational actions that is related to diversity from inclusion.
\end{abstract}

Keywords: Teacher training, inclusion, person with disability, didactic sequence 


\section{Introduction}

La educación es un fenómeno social que permite el progreso de sociedades y contribuye a la generación de conocimiento, una de las instituciones que es señalada como un lugar en que se crea y recrea de manera permanente el conocimiento es la universidad, debido a la generación de teorías, modelos educativos, competencias, pedagogías y desarrollo profesional, pero, sobre todo de ellas debe surgir innovación (Delors, 1996). Lo anterior, señala la idea de que la educación universitaria debe aportar calidad y con ello, cumplir con el propósito crear y formar profesionistas que favorezcan al desarrollo de la sociedad, cultura, ciencia y artes; por lo cual, se resalta la función del docente, debido a que en él recae fomentar competencias, mediante la generación de prácticas educativas innovadoras, con la finalidad de una educación para todos que atiendan las diferencias de los estudiantes (Márquez-Domínguez, Gutiérrez-Barroso \& González-Herrera, 2018; Moreira, Romero \& Torres, 2019; Sagastizábal, 2000).

De tal manera este trabajo busca la innovación en prácticas educativas, a través de la formación docente y desarrollo de competencias necesarias en la búsqueda de una mejor respuesta educativa a los estudiantes. Por ello, se orientó al diseño de estrategias educativas para la atención de estudiantes con discapacidad en la universidad a través de SD basadas en El Modelo para el Desarrollo y Evaluación de Competencias Académicas (M-DECA), el docente vivió un proceso de formación que incluyó dicho modelo y aspectos relevantes sobre inclusión educativa.

El programa de formación se basó en M-DECA de Guzmán, Marín e Inciarte (2014), en el cual se plantea, que ante la necesidad educativa, de afrontar adecuadamente los retos derivados de la sociedad del conocimiento y las reformas educativas en términos de competencias relevantes para el individuo; este modelo busca el diseño, intervención en el aula, análisis, y evaluación de propuestas educativas vinculadas, entre otros, con procesos de formación y evaluación de profesores (Marín, Guzmán, Márquez \& Peña, 2013).

El M-DECA, plantea dentro de los anhelos pedagógicos la búsqueda permanente una docencia transformada y transformadora, para incidir eficazmente en la promoción del cambio y la mejora sustantiva de las situaciones de vida de las personas que se forman; desde esta perspectiva, resulta comprensible la propensión a adoptar propuestas viables o potencial para concretar los propósitos (Guzmán et al., 2014). Lo anterior, puede ser considerado para formar a docentes competentes en atender a la diversidad estudiantil.

Uno de los aspectos que retoma el M-DECA es la generación de SD que permiten generar un andamiaje en el estudiante, es decir, reconoce establecer una estructura y organización en el manejo de la información 
presentada por el docente, siendo esta una estrategia pedagógica fundamental del este modelo. Tal como lo menciona Díaz-Barriga (2013), la SD es una tarea importante para organizar situaciones de aprendizaje que se desarrollarán en el trabajo de los estudiantes para establecer un clima de aprendizaje. También lo señalan Tobón, Pimienta y Garcia (2010, p. 23):

"Las secuencias didácticas son, sencillamente, conjuntos articulados de actividades de aprendizaje y evaluación que, con la mediación de un docente, buscan el logro de determinadas metas educativas, considerando una serie de recursos. En la práctica, esto implica mejoras sustanciales de los procesos de formación de los estudiantes, ya que la educación se vuelve menos fragmentada y se enfoca en metas".

En este ámbito referencial, el trabajo se orienta a aplicar una estrategia pedagógica, sustentada en los procesos de formación y se generan andamios cognitivos, una vez construidos también pueden ser aplicados en los momentos áulicos con estudiantes.

\section{Formación docente}

En el marco de esta investigación se asume el concepto de formación docente como proceso integral con el uso constante de practica reflexivas, estas consisten en la tendencia a la construcción y apropiación crítica de las herramientas conceptuales y metodológicas para el desempeño profesional; es decir, la formación docente consiste en un proceso permanente y continuo, el cual abarca no sólo las experiencias escolares, sino también la totalidad de la trayectoria de vida de los sujetos que en ella participan (Leiva \& Jiménez, 2012; Soto, 2015); por ello la importancia de contar con elementos necesarios como la atención a la diversidad y la atención a estudiantes con alguna discapacidad (Murillo, Ramos \& Sotelo, 2020).

A propósito de ello Corona (2020), plantea desarrollar nuevos modelos, fortalecer los procesos de formación en los docentes y revisar la pertinencia de su oferta, así como cumplir con la necesidad de formarlos en una cultura de diversidad que fundamente la educación inclusiva, su concepción y sus prácticas didácticas. Ante la diversidad, han de trasladarse del alumno al contexto; en lugar de fijarse en las peculiaridades del alumno, para que el centro educativo sea capaz de responder a las necesidades de los estudiantes (Arnaiz \& Caballero, 2020; Durán \& Giné, 2011). Este cambio de paradigma requiere de un esfuerzo notable.

Lo anterior, es uno de los desafíos que hoy enfrenta el docente universitario, es decir, llevar a la práctica la inclusión educativa, implica la realidad compleja que va desde la encrucijada de la segregación encubierta del alumnado con necesidades educativas especiales, hasta la falta de profesionales especializados, así como la escasa formación en educación 
inclusiva de todo el profesorado y la comunidad educativa (Leiva \& Jiménez, 2012). En el ámbito de la educación superior, implica un sinnúmero de cuestiones a atender dentro de la práctica, tales como: la planificación de la enseñanza, la interacción educativa dentro del aula, y la evaluación; acompañado de una actividad dinámica y reflexiva.

Un buen profesor no sólo requiere el dominio de los contenidos de la materia, sino desarrollar habilidades pedagógicas, una capacidad perceptiva y de atención sistemática sobre los avances de sus alumnos para averiguar si están aprendiendo o no y el por qué; qué es lo que les ayuda a aprender (Zambrano, 2005). Para poder atender esta tendencia hacia la inclusión educativa en beneficio de los alumnos, hace falta tener una mayor formación pedagógica y dominio de competencias docentes.

Guzmán et al. (2014), mencionan que el docente universitario es un intelectual capaz de analizar el proyecto institucional; y, en función de ellos, realiza una transposición didáctica, esto es reconocido como un intelectual transformativo, que analiza su práctica educativa, con el fin de crear espacios donde todos tengan las mismas posibilidades, tanto dentro de la escuela como fuera de ella.

La prácticas educativas que el docente puede utilizar para eficientar el proceso de enseñanza, la incorporación de los principios de la pedagogía de la integración y la construcción que se hace de SD, es parte de esta noción donde desarrollan situaciones problema para movilizar saberes y tener una guía formativa que le permite visualizar las intenciones formativas, la utilización de dispositivos de formación, donde se pueda plasmar las competencias a desarrollar; es la SD, el conjunto de actividades de aprendizaje organizadas, estructuradas y articuladas con base en situaciones de integración que las desencadenan y que presentan evidencias de los desempeños de los estudiantes como una forma de facilitar la evaluación de competencias tanto por los profesores como por los estudiantes (Guzmán et al., 2014; Roegiers, 2010), he aquí el desafío que la escuela inclusiva propone a la formación de los docentes.

\section{Inclusión de estudiantes con discapacidad}

La incursión de estudiantes con alguna discapacidad a las aulas universitarias ha tomado por sorpresa a la institución, pues profesores que deben atender a estos alumnos, muchas veces no cuentan con la preparación adecuada o las herramientas teóricas y metodológicas para hacer frente al reto de formar personas con las características que demanda hoy la educación inclusiva (Moreira et al., 2019).

Las actuales transformaciones en las universidades son importantes en la atención socioeducativa recibida por las personas con discapacidad. En el ámbito universitario, la presencia de estudiantes con discapacidad en las aulas ha aumentado de manera considerable, en el caso de la Universidad Autónoma 
de Chihuahua (UACH), se cuenta con una población con discapacidad de 222 estudiantes de pregrado y 9 de grado, ante esta necesidad, se asume como el principal reto la formación docente ante una transformación de las políticas, prácticas y cultura que logren la participación e inclusión educativa dentro los modelos educativos universitarios en México (UACH, 2016).

Hablar de inclusión educativa trasciende el ámbito de la atención a poblaciones con discapacidades, pasando por una diversidad cultural, ideológica, con ello se amplía la esfera de acción a sectores tradicionalmente alejados de la educación, en nivel superior no sólo se limita a crear las condiciones para atender a estudiantes con ciertas discapacidades sino ampliar el abanico de posibilidades que promuevan la incorporación de estudiantes de diversos sectores, su permanencia en los estudios, su egreso y lo más importante abonar a su futuro profesional (Leiva \& Jiménez, 2012; Valenciano, 2009).

Por lo tanto, el acceso a los estudios superiores debería estar basados en: la capacidad, los esfuerzos, la perseverancia, la determinación de los aspirantes y en la perspectiva de la educación a lo largo de toda la vida, tomando debidamente en cuenta las competencias adquiridas anteriormente. En consecuencia, en el acceso a la educación superior no se puede admitir ninguna discriminación fundada en la raza, el sexo, el idioma, la religión, ni en consideraciones económicas, culturales o sociales, ni en incapacidades físicas y brindar una adecuada equiparación de oportunidades (GonzálezGonzález, Gutiérrez-Barroso, González-Herrera \& Márquez-Domínguez, 2017).

La Convención sobre los Derechos de las Personas con Discapacidad, la Organización de Naciones Unidas (2006), apuntó la obligación de asegurar que las personas con discapacidad accedan -sin discriminación y en igualdad de condiciones al resto- a la educación superior, a la formación profesional, a la educación para adultos y al aprendizaje a lo largo de la vida (artículo 24.5, bajo el título Educación).

De igual forma, mejorar el acceso, la promoción y el egreso académico de colectivos vulnerables en entornos de riesgo de exclusión social en la región, se sitúa en este marco contextual, ha servido para analizar el contexto de referencia identificar aquellos colectivos que presentan necesidades específicas y analizar los programas y medidas adoptadas por las instituciones iberoamericanas de educación superior para asegurar su acceso y permanencia (Hernández \& Valcárcel-Resalt, 2016). Así como se establece en el Artículo 12 del capítulo III sobre Educación de la Ley General para la Inclusión de las Personas con Discapacidad, el impulsar la inclusión de las personas con discapacidad en todos los niveles del sistema educativo nacional y promover el derecho a la educación de las personas con discapacidad, prohibiendo cualquier discriminación en planteles, centros educativos, guarderías o del 
personal docente o administrativo del Sistema Educativo Nacional (Diario Oficial de la Federación, 2018).

Por lo anterior, en esta investigación se orienta a la formación del docente, para desarrollar prácticas educativas que favorezcan los procesos de inclusión educativa; esto implica que el estudiante logre acceder a los aprendizajes, elementos y saberes al igual que cualquier otro alumno (Garrote, Jiménez \& Palomares, 2015). Así como el sentirse parte de la comunidad estudiantil; esto exige a los docentes desempeñen múltiples funciones, una de ellas, es el diseño de estrategias didácticas innovadoras, practicas reflexivas y nuevos mecanismos de formación que elimine las barreras de participación y aprendizaje (Chávez, Balderrama \& Figueroa, 2015; Paz-Maldonado, 2020).

\section{Método \\ Participantes}

Participaron diez docentes que imparten clase en grupos que tienen estudiantes con discapacidad (visual y motriz); a los cuales se les formó con un programa formativo, con el objetivo de valorar las experiencias vividas, en proceso de formación docente orientado al desarrollo de prácticas educativas inclusivas.

El estudio adopta un enfoque metodológico donde se sitúan de manera concreta varias tradiciones metodológicas pertenecientes al paradigma cualitativo: la primera se refiere al estudio de casos (Stake, 2010; Yin, 1994), la segunda se ubica como una investigación-acción participativa (DíezGutiérrez, 2020; Elliott, 1990; Kemmis \& McTaggart, 1988). La investigación-acción participativa es un proceso crítico. Esta metodología de formación docente debe ser guiada por procesos reflexivos que busca transformaciones críticas y éticas (Barba, 2019).

Se utilizan diversas técnicas e instrumentos para la recolección de la información, prevaleciendo los grupos de discusión, diálogos reflexivos y se plasma los criterios de validación y revisión de secuencias.

\section{Unidades de análisis}

Programa de formación docente: conjunto de actividades didácticas y pedagógicas basadas en el M-DECA, denominado "Intervenir e investigar en el aula" la atención de alumnos con discapacidad, el cual permitió instruir a los docentes participantes.

\section{Unidades de observación}

Experiencias vividas en la formación docentes: son aquellas narrativas reflexivas que permite analizar y comprender situaciones referentes al proceso de enseñanza, detecta situaciones en las cuales puede contribuir e intervenir de manera oportuna para la mejorar en la enseñanza. 


\section{Herramientas/Instrumentos}

Observación participativa: cuyo propósito es la obtención de datos acerca de la conducta a través de un contacto directo y en términos de situaciones específicas en las cuales sea mínima la distorsión producida en los resultados a causa de la implicación del observador; se trata de una observación participativa dado que el observador forma parte del contexto comprometiéndose en las actividades que empieza a observar (Gutiérrez, 2006; Sánchez, 2009).

Videograbación de la práctica: es un instrumento que permite al investigador a acercarse a los acontecimientos de la vida real tal como ocurren naturalmente, es decir, sin presencia del investigador, se puede observar la misma situación en varias ocasiones incluso se pueden observar.

Diálogo reflexivo mediante el trabajo de tríadas: se trabaja de manera participativa; privilegiándose el trabajo colaborativo y de práctica reflexiva, que se maneja en tres modalidades: la reflexión personal, en "díadas" y en "tríadas"; esta organización del trabajo grupal resulta importante en la construcción del "proyecto formativo" que los docentes diseñarán y aplicarán en su intervención (Guzmán et al., 2014).

\section{Procedimiento}

Se inició la revisión de literatura para poder integrar el M-DECA y las competencias de programa formativo, así como todo lo concerniente a la inclusión de estudiantes con discapacidad al proceso de diseño, posteriormente el desarrollo los módulos y la validación del programa formativo para docentes denominado intervenir e investigar en el aula: alumnos con discapacidad, seguido se convocó a los docentes para su participación de forma voluntaria, una vez finalizada la formación se documentaron las experiencias de este proceso formativo.

\section{Resultados}

Se muestran resultados relevantes referentes a la formación docente para el diseño y contenidos del programa de formación de profesores, a través de las experiencias y narrativas generadas por diálogos reflexivos. El proceso de formación docente forjó proyectos formativos inclusivos, como una estrategia pedagógica que contribuye a la formación integral.

Para ofertar este programa de formación docente se obtuvo la validación académica por las instancias directivas de la UACH; por ello, el programa fue diseñado bajo la modalidad de diplomado comprendiendo 100 horas de dedicación distribuidas en cuatro módulos. Dicha elaboración fue basada en el M-DECA, que busca el diseño, intervención en el aula, análisis y evaluación de propuestas educativas vinculadas, entre otros, con procesos de formación y evaluación de profesores. 
El programa de formación fue construido para el desarrollo de competencias docentes orientadas a una práctica docente incluyente que los lleve a planificar y diseñar alternativas innovadoras tendientes al establecimiento de mejores prácticas en la escuela. Las competencias que conforman el perfil competencial universitario en la UACH y que fueron desarrolladas: Procesos de transposición didáctica; Diseño de una docencia innovadora; Coordinación de la interacción pedagógica inclusiva; Gestión de la progresión de los aprendizajes; y, Valorar el desarrollo de las competencias.

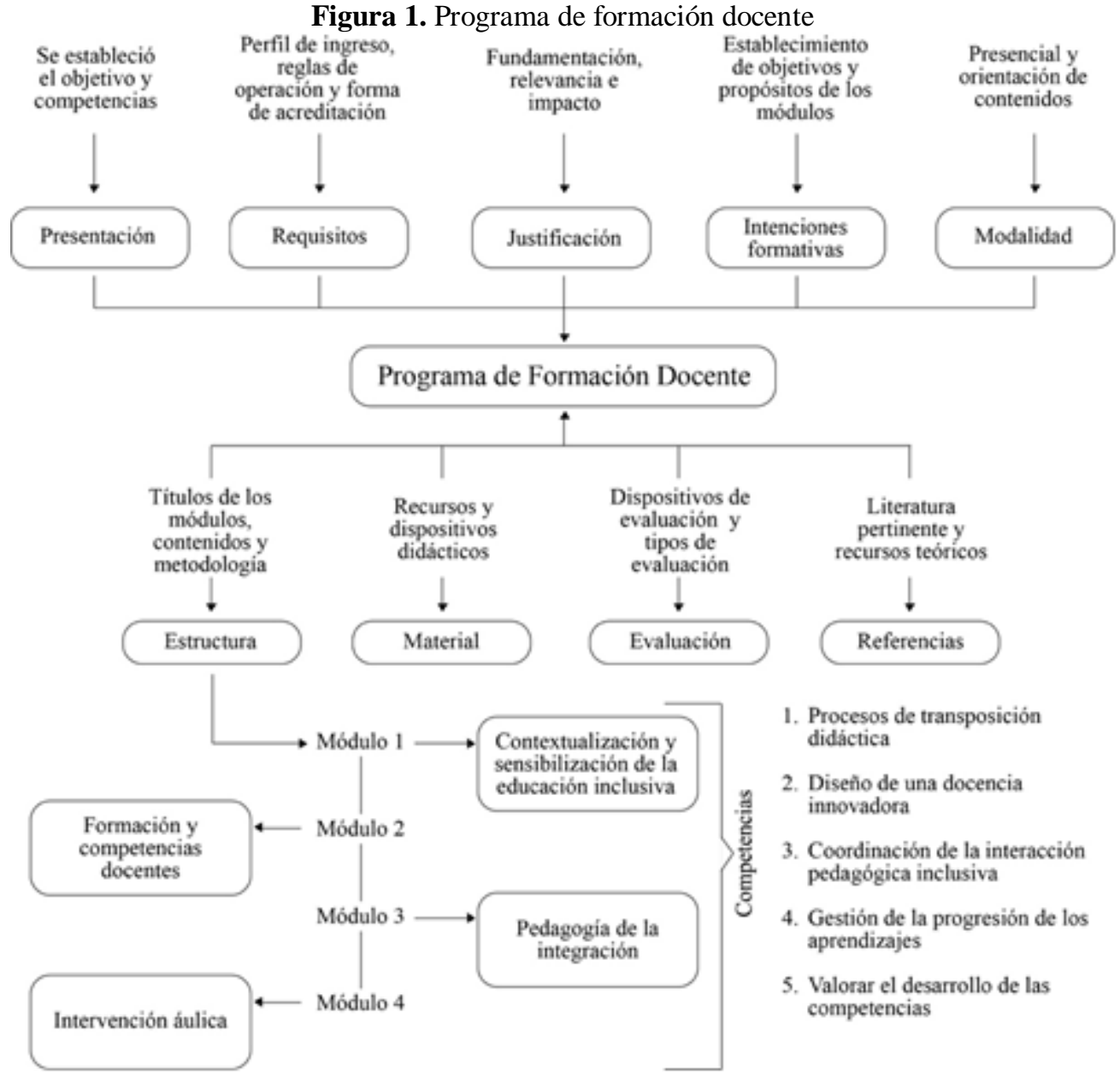

En la Figura 1, se muestra en la parte superior y media los requisitos que se tuvieron que contemplar para la validación académica; además, en la parte inferior se presenta la estructura y los elementos del programa de formación docente, así como los nombres de los módulos del contenido y las 
competencias a desarrollar en cada uno. Resultado de la implementación de estos módulos se presentan los momentos relevantes en la formación y de las experiencias vividas por los docentes participantes:

\section{Resultados del proceso de formación docente}

Se observó en el primer momento del programa de formación, que los docentes fueron participativos y receptivos ante la formación y el gran interés por ofrecer alternativas inclusivas para los estudiantes; quienes ya tenían experiencia con alumnos con discapacidad se distinguían confiados e interesados, contrario a una de las docentes quien nunca había tenido alumno con discapacidad, se mostró renuente y callada en cuanto e incluso ella comenta que si pudiera no escogería tener un alumno con estas condiciones.

Se planteó al grupo una situación problema mediante el siguiente cuestionamiento: ¿Por qué estamos en este programa? ¿Acaso sabes tú porque estás aquí? ¿He sido excluido o he excluido? ¿Qué es la discapacidad? ¿Cuento con las competencias para trabajar con alumnos con discapacidad? al responder estos cuestionamientos surgieron más cometarios acerca de la necesidad de formación a los docentes universitarios sobre las prácticas inclusivas. La mayoría de los participantes expresaron no tener ni idea de cómo trabajar con alumnos con discapacidad y los que han trabajado, su experiencia ha sido complicada, el poderlos integrar en las clases sin estar preparados para esta situación. Mientras tanto, un docente con experiencia, en el ámbito de la discapacidad enfatizó:

...cada alumno presenta características distintas y por lo tanto aprenden a diferentes ritmos, lo cual nos obliga a modificar o adecuar la metodología tradicional, en ocasiones los objetivos, los instrumentos de evaluación, incluso contenidos, tenemos que utilizar más herramientas y medios para eliminar esas barreras que impiden regularizar ese ritmo de aprendizaje, algo curioso es que aún existen ciertas costumbres en maestros que se resisten a estos cambios, desde mi punto de vista se requiere más trabajo en la concientización y preparación; sobre todo un buen inicio es el cambio de actitud hacia estos alumnos... (Docente 1).

Se asintió que en las actividades diarias, de manera consciente o inconsciente, se presenta la exclusión; expresado en una historia de vida en el plano personal de una docente al ser sensibilizada, señaló:

... dentro mi historia de vida en la que puedo relacionar el tema de cómo he vivido la exclusión o la inclusión, tiene que ver con la siguiente historia fui excluida por las niñas de la colonia de mi edad, por cómo era o fui educada y cómo me dirigía a los demás. En ocasiones me integraban al juego y en otras no, simplemente porque 
no querían que jugara, eso me hizo sentir mal el no ser parte de algunos juegos o actividades solo porque ellas me percibían diferente a ellas... el recodar esto me hace reflexionar sobre cómo podría sentirse un estudiante o cualquier persona con discapacidad al no sentirse integrado... (Docente 3).

En el segundo momento de la formación docente, consistió en desarrollar el Módulo 2, donde se trabajó los modelos de formación y las competencias docentes, en las cuales se pretendió reflexionar sobre su práctica educativa y su postura epistemológica sobre la trasposición de los saberes, donde se mostró que los docentes no conocen prácticas educativas tendientes a la inclusión y que ellos pensaban de manera generalizada; que había que adecuaciones curriculares y que era complicado pues ellos no sabían como adecuar la clase según la discapacidad, solo dos de los docentes, quienes si tenían experiencia de haber trabajado con alumnos con discapacidad, comentaban alternativas para el trabajo con ellos y que los cambios no tenían que ser significativos.

El que se trabajara de manera colaborativa, en comunidades de practica y a través de tríadas, en donde los participantes tuvieran asignado un rol dentro de ella; resultó ser algo que a los docentes se les quedó muy presente del programa de formación docente. Una de las actividades de aprendizaje fueron las comparaciones sobre las prácticas educativas que favorecen la inclusión y el abordaje de competencias docentes; las cuales fueron elaboradas tomando en cuenta aspectos inclusivos en cada dominio. Este ejercicio permitió generar una opinión homogénea sobre la necesidad de desarrollar competencias docentes, como se muestra en la figura 2.

Figura 2. Principales respuestas de los docentes sobre las competencias

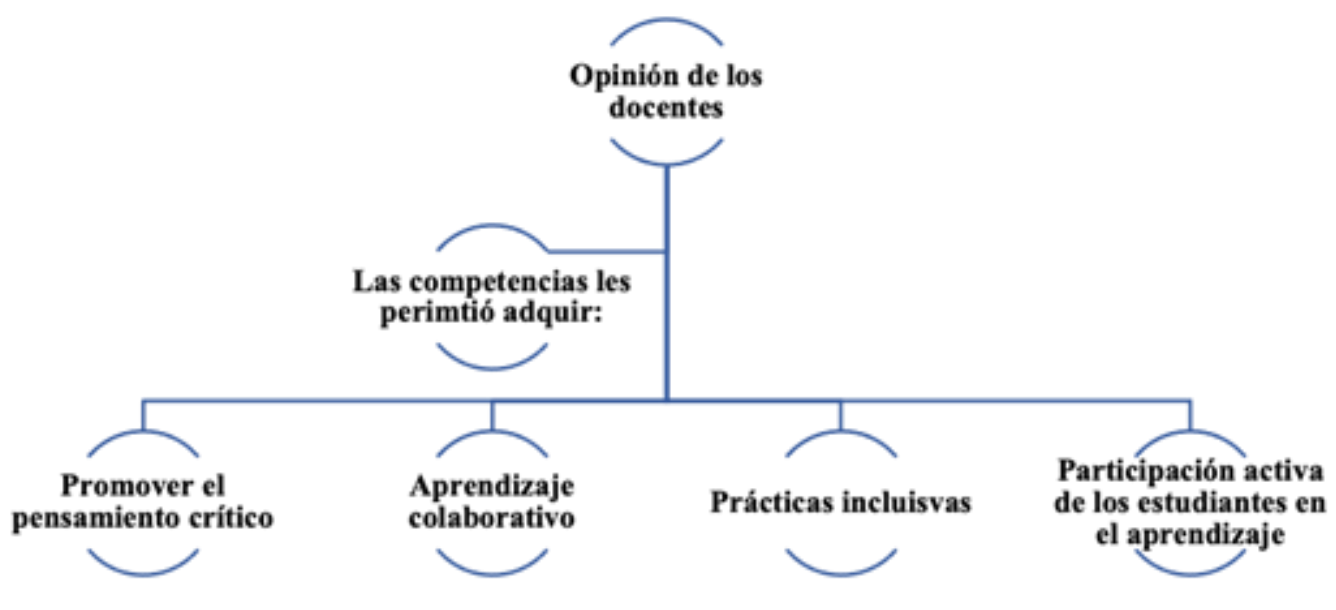


Para el tercer momento del proceso de formación los docentes entregaron evidencias de desempeño, donde el papel que juega el aprendizaje colaborativo como mecanismo, para generar inclusión dentro aula es indispensable. Las premisas reflexionadas sobre ello, aporta para la inclusión, la interacción entre los pares; construcción activa y conjunta de los conocimientos; estudiantes y docentes mediadores; andamios o ayudas a las necesidades de otros estudiantes; el énfasis en el intercambio de experiencias; y, generación de saberes compartidos.

El ver el aprendizaje colaborativo, como un generador de sistemas educativos inclusivos, que favorece y promueve el diálogo, la convivencia, los de apoyos entre los propios estudiantes a fin de alcanzar una meta en común... (Docente 2) ...permite la convivencia, las de tareas compartidas y la empatía pues al compartir experiencias y condiciones de vida son cosas indispensables para caminar hacia una educación más inclusiva... (Docente 4).

Una de las evidencias de desempeño, posterior a la transposición de las competencias docentes; fue el diseño del proyecto formativo constituido por SD, de acuerdo con el M-DECA que permean el análisis de la práctica educativa transitando comparativamente del momento del diseño de la docencia al de la intervención en el aula. Estos diseños fueron valorados por el autor de dicho modelo. Se mostraron proyectos formativos a los que no fue necesario hacer ningún cambio, como en el caso de "Síndromes y trastornos" al tomar en cuenta una discapacidad motriz (amputación de brazo). Para este proyecto, se le indicó:

La situación problema, es innovadora pues al ser solo una imagen promueve en el alumno pueda imaginar y hacer inferencia sobre el tema que se va a desarrollar, antes de que el docente explique de qué se trata. Muy bien las actividades, las evidencias están de acuerdo con las actividades de aprendizaje...

En otro caso, la situación problema consistió en un video sobre una entrevista de una persona con burnout; las sugerencias fueron que se convirtiera en audio, ya que lo que importaba era lo que se decía y no lo que se veía y aunque el alumno con discapacidad visual si podía entender el video, porque tenía sonido se comentó que el presentar mejor un audio sería una actividad inclusiva para él, en igualdad de condiciones que para los demás compañeros del grupo. Para otro proyecto, se recomendó incorporar la tríada, herramienta metodológica para el trabajo en grupo, dónde el alumno con discapacidad estuviese con compañeros proactivos y sociales, así de esta manera el alumno con discapacidad podría impulsar su desenvolvimiento aún con Asperger. Además, el experto señaló: 
...recuerden que las situaciones problema poseen una importancia medular y están centradas en motivar y captar la atención del alumno...

Por último, el proyecto donde se abordarían los riesgos de la actividad física; se le sugirió presentar un caso real, donde puedan todos los alumnos reconocer y analizar el riesgo de un paciente/cliente desarrolle un problema durante la práctica de una sesión de ejercicio físico y poner en marcha pautas para minimizar los riesgos y la necesidad de un plan escrito organizado que perfile cómo se puedan manejar diferentes niveles de problemas relacionados con el ejercicio físico en un centro deportivo. Además, se le recomendó, el trabajo en tríadas para la identificación de las características del alumno con discapacidad visual y ver cuál papel de la tríada podría realizar con su desempeño, destacando las fortalezas con el rol de presentador, pues se le facilitaba exponer y hablar.

Estas son algunas de las observaciones que permitieron a los docentes mejorar sus SD e incluir otras actividades fundamentadas por el M-DECA y hacia la inclusión educativa. Después de la revisión de las SD, se adaptaron las recomendaciones para la versión final y así realizar la implementación.

\section{Resultados de las experiencias de los participantes}

La implementación de los diálogos reflexivos y el trabajo de tríadas; se generó las narrativas de experiencias docentes al planteamiento de dos preguntas generadoras. La primer sobre la apreciación del programa de formación:

... mucha información, aprendí cosas nuevas e interesantes y pues la experiencia de la discapacidad me gusto, pero me hubiera gustado que nos dieran más estrategias para trabajar con los alumnos... (Docente 4).

... en general creo que el aprender usar el andamio ayuda mucho a organizar las clases. También el haber trabajado en tríadas me agrado, aunque creo que eso les quito un poco de responsabilidad a otros compañeros que no tenían experiencia en discapacidad y a la vez creo que la mía les facilito el trabajo... (Docente 2).

...Considero que el programa de formación estuvo bien, mucha información y buena experiencia, el conocer cómo se sentían algunos de los compañeros respecto a la discapacidad y sobre su conocimiento o desconocimiento me llamo la atención, pues yo en dos ocasiones ya había tendió alumno con discapacidad, por lo que buscaba ya estrategias para trabajar con él; en general me pareció bien... (Docente1). 
...Me pareció bien en general, muchos temas debieron ser amas afondo, con la inclusión de los alumnos con discapacidad en la universidad, se visualizó un cambio de actitud primordial para empezar a dar una atención adecuada a estos alumnos y es verdad que no se vieron estrategias específicas para discapacidad, pues de los que se trata es generar estrategias para todos... (Docente 3).

En cuanto a la segunda pregunta generadora de ¿cómo fue la experiencia de participar en esta investigación, con el reto de ser videograbado en la intervención del proyecto formativo diseñado en una clase?

...Me dio pánico, fue un reto ante la posible critica, mi grupo se portó muy bien sin hablar, de echo se portaron mejor y todo fluyo, pero definitivamente el ser observados por otro compañero, no es sencillo y más si vas a probar cosas que no has hecho antes... (Docente 8).

...El que voy a hacer, preparar algo diferente donde integra al alumno, el que hay ciertas cosas que se pueden hacer, adecuar para incluirlo y sentí que fue un reto y enfrentarme a un miedo, que aún lo tengo un poco, pero fue una grata experiencia. Estrategia de intervención innovadora, con una función esencial en mi organización didáctica, que me mis alumnos lo conozcan cómo está estructurada la sesión, ser mi propio observador de mi docencia o sistematizar, autoevaluarme... (Docente 2).

...Concientización hacer consiente. El ser observador ayuda a mejorar nuestro desempeño, además de profundizar más en ciertos temas y actividades, funciones de la triada como aprendizaje. No hay un libro sino la actitud, se me queda el aprendizaje... (Docente 1).

\section{Resultados de vivir mi experiencia}

Por último, comparto los resultados de vivir mi experiencia; puesto que al realizar una investigación acción participativa, durante del desarrollo de este estudio, tuve una participación sui generis, al jugar tres roles: 1) como investigadora, 2) como diseñadora y formadora del programa, 3) como parte de un proceso formativo participé como "estudiante", como producto de los tres módulos de formación, logré construir mi "proyecto formativo" para desarrollar y evaluar competencias en mis estudiantes:

...Durante la experiencia en el proceso de formación, fueron un sinnúmero de aprendizajes los que viví durante la aplicación de los módulos del programa, el que compañeros docentes se convirtieran en mis estudiantes y al mismo tiempo yo formarme dentro de ese mismo programa de formación, al presenciar las participaciones de los otros miembros del equipo de formación, quienes lograron realizar una transposición didáctica invaluable para asimilación del M-DECA y 
las competencias docentes propuestas para este dispositivo de formación...

...Fue una experiencia significativa, puesto que había docentes con nula experiencia en la atención a la inclusión y discapacidad y otros con mayor acercamiento y experiencia en ello. Por ello, me permitió que las prácticas de los más experimentados enriquecieran los conocimientos de los otros...

\section{Discusiones y conclusiones}

Dentro del objetivo de este trabajo fue presentar las narraciones de las experiencias resultadas de la generación de un programa formativo para docentes, el cual pretendió buscar en el docente prácticas reflexivas y colaborativas que se vieran plasmadas en el diseño de las SD, para fomentar estrategias y prácticas educativas inclusivas para estudiantes con discapacidad, lo cual permitió visualizar en los resultados que los docentes lograron formarse a través de la reflexión que realizan sobre su práctica, aspecto primordial en el proceso de formación docente, se apreciaron las experiencias que promovieron la participación y reflexión crítica, consiguieron comprender criterios del M-DECA y adoptarlos en sus estrategias, estos descritos en las matrices donde se constata los elementos que el docente incluyó en la SD; al igual que en el intercambio de experiencias que promueven reflexión y análisis; espacios donde se favorece la deconstrucción de las prácticas docentes, tal como lo señala Agreda y Pérez (2020).

De igual forma, cabe resaltar que el programa fomentó la participación activa del docente plasmando en las SD, el manejo del trabajo colaborativo entre estudiantes con y sin discapacidad, como en el estudio de Guerra, Rodríguez y Artiles (2019), quienes señalan que el rol del docente debe promover una función de guía y gestor de aprendizaje, para fomentar la colaboración e integración entre iguales que favorezcan el proceso de aprendizaje, exige de una buena programación docente. Para ello, el profesorado debe crear un escenario donde se fomente tanto el aprendizaje personal como el aprendizaje de equipo; aparte, con la finalidad de dotar a sus estudiantes de los recursos y los medios necesarios para el logro de los objetivos de aprendizaje, y esto se pudo apreciar en los resultados presentados ya que en los criterios de las secuencias y en proceso de formación del programa están establecidos para que se lleven a cabo para la aplicación de los docentes.

Además, de que la integración a las prácticas educativas, dentro de las SD se vio reflejada la perspectiva de inclusión, en las cuales notan aspectos que les permiten hacer ajustes, incluir situaciones problema, utilizar dispositivos de evaluación más eficientes y sobre todo el promover el trabajo colaborativo; así como visualizar las características de los estudiantes con y 
sin discapacidad. Pues el aprendizaje cooperativo constituye una metodología eficaz para desarrollar el sentido crítico y de tolerancia, trascendiendo lo estrictamente académico y facilitando la práctica de hábitos de cooperación, solidaridad y trabajo en grupo y se caracteriza por el principio de igualdad (León del Barco \& Latas, 2019).

Todo esto con un acercamiento a la inclusión de estudiantes en el ámbito universitario contribuyendo a la exigencia de mirar a la diversidad respetando las diferencias y garantizando la plena participación en todos los procesos educativos (Benet-Gil, 2020).

Diversos estudios previos, señalan que los modelos de inclusión en la educación superior, analizando las diferentes actuaciones a favor de los estudiantes con alguna discapacidad desde implantación de accesos adaptados, adecuaciones a procesos de ingreso y permanencia, campañas de sensibilización y claramente prácticas educativas inclusivas (Cruz \& Casillas, 2017; Díez-Rodríguez, 2020; Flores, García \& Romero, 2017).

En la actualidad se observan cambios relevantes en la configuración de las instituciones educativas y los participantes de estos. Un gran número de personas tradicionalmente excluidas del sistema educativo regular, han ingresado a éste, contradiciendo a la idea de que las aulas estaban constituidas por un grupo homogéneo de alumnos en cuanto a sus habilidades, raza, género, lenguaje, estructura familiar y discapacidad, entre otros. Por lo tanto, esta nueva configuración de las instituciones educativas implica una diversidad en los sujetos que las habitan. Una de las respuestas a esta diversidad ha sido un llamado desde organismos internacionales y estamentos gubernamentales a transformar las escuelas en comunidades más inclusivas (Infante, 2010).

Se puede concluir que los programas de formación como éste, el cual implica una vasta revisión de literatura en relación con la formación docente en temáticas sobre la sensibilización ante la discapacidad, estrategias tendientes a la inclusión educativa; busca mejorar las prácticas de una universidad que requiere atender de manera pertinente y oportuna la diversidad de sus estudiantes.

Esto observó una participación activa de los docentes, los cuales en su experiencia de formación comentan la utilidad y la reflexión que les proporcionó el programa, es decir, se sensibilizaron en el tema de la inclusión, primer paso hacia la trasformación de las prácticas educativas. Además, las $\mathrm{SD}$, permiten concretar contenidos adecuados y actualizados que sirvan de guía al docente para su propia generación de dispositivos de formación en el aula, en la atención de las necesidades de los alumnos que presentan una discapacidad, adquiriendo las competencias docentes necesarias para que se practique una docencia que le permita construir un proyecto formativo inclusivo en el desarrollo de sus estudiantes. 
Siendo así, que la formación docente constituye un desafío para las instituciones de educación superior destacando la necesidad profesional que lidere las acciones educativas relacionadas con la diversidad desde la inclusión y desde abrir espacios de discusión teórica con una mirada crítica a los cursos de formación docente.

\section{References:}

1. Agreda, A. \& Pérez, M. (2020). Relación entre acompañamiento pedagógico y práctica reflexiva. Espacios en Blanco. Revista de Educación, 2(30), 219-232.

2. Arnaiz, P. \& Caballero, C.M. (2020). Estudio de las aulas abiertas especializadas como medida específica de atención a la diversidad. Revista Internacional de Educación para la Justicia Social, 9(1), 191 210. https://doi.org/10.15366/riejs2020.9.1.009

3. Barba, M. (2019). La investigación-acción participativa desde la mirada de las maestras participantes en un proceso de formación permanente del profesorado sobre educación inclusiva. (Tesis doctoral publicada).

4. Benet-Gil, A. (2020). Desarrollo de políticas inclusivas en la educación superior. Convergencia Revista de Ciencias Sociales, [S.l.], 27, 1-31. https://doi.org/10.29101/crcs.v27i82.11120

5. Chávez, J.A., Balderrama, J.A. \& Figueroa, S. (2015). Análisis de necesidades de formación docente para la inclusión educativa en el Nivel Superior. Memorias del Encuentro Internacional de Educación a Distancia, 4(4), 1-15.

6. Corona, M.M. (2020). Formación docente en la educación superior privada. Reflexiones desde tres dimensiones de tensión: la social, una técnica y la formativa-mística Revista sinergias educativas. 5(1), 412432. doi: https://doi.org/10.37954/se.v5i1.75

7. Cruz, R. \& Casillas, M.A. (2017). Las instituciones de educación superior y los estudiantes con discapacidad en México. Revista de la Educación Superior, $41(181)$, 37-53. https://doi.org/10.1016/j.resu.2016.11.002

8. Delors, J. (1996). La Educación encierra un tesoro. España: UNESCO.

9. Diario Oficial de la Federación (2018). Ley General para la inclusión de las personas con discapacidad. México: SEP. http://www.diputados.gob.mx/LeyesBiblio/pdf/LGIPD_120718.pdf

10. Díaz-Barriga, A. (2013). Secuencias de aprendizaje. ¿Un problema del enfoque por competencias o un reencuentro con perspectivas didácticas? Revista de Curriculum y formación del profesorado. 17 (3) 11-33. https://recyt.fecyt.es/index.php/profesorado/article/view/41685 
11. Díez-Gutiérrez, E.J. (2020). Otra investigación educativa posible: investigación-acción participativa dialógica e inclusiva. Márgenes, Revista de Educación de la Universidad de Málaga, 1 (1), 115-128. doi: http://dx.doi.org/10.24310/mgnmar.v1i1.7154

12. Durán, D. \& Giné, C. (2011). La formación del profesorado para la educación inclusiva: Un proceso de desarrollo profesional y de mejora de los centros para atender la diversidad. Revista Latinoamericana de Educación Inclusiva, 5(2), 153-170.

13. Elliott, J. (1990). La Investigación-Acción en Educación. España: Morata.

14. Flores, V., García, I. \& Romero, S. (2017). Identificación de prácticas inclusivas de docentes formadores de docentes. Liberabit, 23(1), 3956. https://10.24265/liberabit.2017.v23n1.03

15. Garrote, D., Jiménez, S. \& Palomares, A. (2015). Análisis de las dificultades de aprendizaje en estudiantes con discapacidad intelectual. European Scientific Journal, ESJ, 11(25), 1-16. http://eujournal.org/index.php/esj/article/view/6199

16. González-González, D., Gutiérrez-Barroso, J., González-Herrera, A.I. \& Márquez-Domínguez, Y. (2017). Inserción Laboral De Personas Con Discapacidad. European Scientific Journal, ESJ, 13(29), 109. https://doi.org/10.19044/esj.2017.v13n29p109

17. Guerra, M., Rodríguez, J. \& Artiles, J. (2019). Collaborative learning: innovative experience with university students. Revista de estudios y experiencias en educación, 18(36), 269-281. doi.org/10.21703/rexe.20191836guerra5

18. Gutiérrez, R. (2006). Los estudios de casos una opción metodológica para investigar la educación artística. En Martín, R. (2006). Investigación en educación artística. Granada: Universidades de Granada y de Sevilla.

19. Guzmán, I., Marín, R. \& Inciarte, A. (2014). Innovar para Transformar la Docencia Universitaria. Modelo para la Formación Competencias. Venezuela: Universidad del Zulia.

20. Hernández, M.Á. \& Valcárcel-Resalt, T. (2016). Éxito académico de colectivos vulnerables en entornos de riesgo en Latinoamérica. Revista Latinoamericana 15(45), 252.

21. Infante, M. (2010). Desafíos a la formación docente: Inclusión educativa. Pontificia Universidad Católica de Chile. Estudios $\begin{array}{lll}\text { Pedagógicos 36(1), 287-297. } & \text { 36 }\end{array}$ http://mingaonline.uach.cl/pdf/estped/v36n1/art16.pdf

22. Kemmis, S. \& McTaggart, R. (1988). Cómo Planificar la Investigación-Acción (R. G. Salcedo, Trad.). Laertes. 
23. Leiva, J.J. \& Jiménez, A. (2012). La educación inclusiva en la universidad del siglo XXI: Un proceso permanente de cambio. $\begin{array}{llll}\text { Investigación } & y & \text { Docencia } & \text { (8), }\end{array}$ http://www.revistareid.net/revista/n8/REID8art3.pdf

24. León del Barco, B. \& Latas, C. (2007). La formación en técnicas de aprendizaje cooperativo del profesor universitario en el contexto de la convergencia europea. Revista de Psicodidáctica, 12(2), 269-277. https://ojs.ehu.eus/index.php/psicodidactica/article/viewFile/224/220

25. Marín, R., Guzmán, I., Márquez, A. \& Peña, M. (2013). La evaluación de competencias docentes en el modelo DECA: anclajes teóricos. Formación universitaria 6(6), 41-54. Doi:10.4067/S071850062013000600005

26. Márquez-Domínguez, Y., Gutiérrez-Barroso, J. \& González-Herrera, A.I. (2018). La Formación Docente: La Metáfora De La Caja Del Formador. European Scientific Journal, ESJ, 14(7), 20. https://doi.org/10.19044/esj.2018.v14n7p20

27. Moreira, T., Romero, S. \& Torres, I. (2019). Intervención del trabajador social en el sistema educativo en personas con discapacidad, Revista Contribuciones a las Ciencias Sociales.

28. Murillo, L.D., Ramos, D., García, I. \& Sotelo, A. (2020). Estrategias educativas inclusivas y su relación con la autoeficacia de docentes en formación. Revista Actualidades Investigativas en Educación, 20(1), $1-25$. doi. 10.15517/aie.v20i1.40060. https://revistas.ucr.ac.cr/index.php/aie/article/view/40060/40671

29. Organización de las Naciones Unidas (2006). Convención Internacional sobre los derechos de las personas con discapacidad. https://www.ohchr.org/Documents/Publications/AdvocacyTool_sp.pd f

30. Paz-Maldonado, E. (2020). Inclusión educativa del alumnado en situación de discapacidad en la educación superior una revisión sistemática. Revista Teoría de la educación, 32(1), 123-146. DOI: 10.14201/teri.20266

31. Roegiers, X. (2010). Pedagogía de la integración. Competencias e integración de los conocimientos en la enseñanza. México: Fondo de Cultura Económica.

32. Sagastizábal, P. (2000). Un Secreto para Julia. Sudamericana.

33. Sánchez, J.F. (2009). Análisis del clima de aula en educación física. Un estudio de casos. (Disertación doctoral). http://www.biblioteca.uma.es/bbldoc/tesisuma/17677907.pdf

34. Soto, M.C. (2015). Experiencias docentes en intervención psicomotriz para el logro de competencias en preescolar. (Tesis Doctoral). Facultad de Ciencias de la Cultura Física, Universidad Autónoma de 
Chihuahua,

Chihuahua, México. http://doctorado.fccf.uach.mx/estudiantes/4.\%20MA.\%20CONCEPC ION\%20SOTO\%20VALENZUELA.pdf

35. Stake, R. (2010). La investigación con Estudio de Casos. Morata.

36. Tobón, T., Pimienta, J. \& Garcia, J. (2010). Secuencias Didácticas: Aprendizaje y evaluación de competencias. Pearson.

37. Universidad Autónoma de Chihuahua (2019). Estadística básica.UACH.

https://uach.mx/assets/media/publications/2017/10/143_agendaestadistica/estadistica-basica-2018-2019.pdf

38. Valenciano, G. (2009). Construyendo un concepto de educación inclusiva: Una experiencia compartida. En Sarto, M.P. y Venegas, M.E. Aspectos clave de la educación inclusiva. (pp.13-24). Publicaciones del INICO. Salamanca, España. Colección Investigación. http://sid.usal.es/idocs/F8/FDO22224/educacioninclusiva.

39. Yin, R. (1994). Case Study Research: Design and Methods (2 ${ }^{\mathrm{a}}$ ed.). SAGE. Thousand Oaks, CA.

40. Zambrano, A. (2005). Conocimiento, saber y pensamiento: una aproximación a la didáctica de las matemáticas. Equis Angulo 1(1), 16. 